\title{
Competencia digital y competencia digital docente: una panorámica sobre el estado de la cuestión
}

\section{Students' and Teachers' Digital Competence: an overview on research status}

\author{
Mercè Gisbert Cervera \\ Universitat Rovira i Virgili \\ Merce.gisbert@urv.cat \\ Juan González Martínez \\ Universitat Rovira i Virgili \\ Juan.gonzalezm@urv.cat \\ Francesc Esteve Mon \\ Universitat Rovira i Virgili \\ Francescmarc.esteve@urv.cat
}

Recibido: $28 / 04 / 2016$

Aceptado: 09/05/2016

Publicado: 30/06/2016

\section{RESUMEN}

La investigación en Tecnología Educativa ha venido desarrollando en los últimos años los conceptos de competencia digital del estudiante y competencia digital docente. El primero tiene que ver con las capacidades necesarias para aprender en la Sociedad de la Información; el segundo, con la necesidad de ser competente en el uso de la tecnología educativa como docente. En relación con ambos conceptos, la investigación aplicada ha avanzado considerablemente en nuestro contexto. Detallamos los principales hitos hasta la fecha: conceptualizaciones, diseño de instrumentos de diagnóstico, desarrollo de rúbricas de evaluación y exploración de posibilidades 3D para el desarrollo de las competencias. Y, por último, se abordan las principales líneas de investigación que deben desarrollarse en el futuro de forma preferente.

\section{PALABRAS CLAVE}

Competencia digital; Maestros en formación; Formación de maestros.

\section{ABSTRACT}

In recent years The research in Educational Technology has been developing the concepts of students' and teachers' digital competence. The concept of digital competence has to do with the skills every person needs to learn in the Information Society; the second one is related to the needs of being proficient in the use of educational technology as a teacher. For both concepts, applied research has made significant progress in our context. We detail the major milestones so far: several conceptualizations, the design of diagnostic instruments, the development of rubrics for assessing these competences and the exploration of the possibilities of $3 D$ technologies for the development of digital skills. And finally, we show the main lines of research to be preferentially developed in the future.

\section{KEYWORDS}

Technological Literacy; Preservice Teachers; Teacher Training.

\section{CITA RECOMENDADA}

Gisbert, M., González, J. y Esteve, F. (2016). Competencia digital y competencia digital docente: una panorámica sobre el estado de la cuestión. RIITE. Revista Interuniversitaria de $\begin{array}{lllll}\text { Investigación en Tecnología Educativa, } & 0, & \text { 74-83. Doi: }\end{array}$ http://dx.doi.org/10.6018/riite/2016/257631 


\section{INTRODUCCIÓN}

Durante los últimos años, una parte de la investigación en Tecnología Educativa se ha centrado en el análisis del conjunto de conocimientos, destrezas y actitudes que los individuos necesitamos para sobrevivir en la Sociedad de la Información (SI). No se trata de una reflexión específica sobre el uso de tales o cuáles tecnologías, ni tampoco sobre la eficiencia de los recursos en el aprendizaje o sobre las pautas que deben seguirse en el momento de su aplicación a los procesos de aprendizaje. Bien al contrario, se trata de un tópico de investigación de tipo mucho más transversal, que parte del cambio que se ha operado en la relación del ser humano con la información y con los medios para acceder a ella, para gestionarla y para transformarla en conocimiento, completamente nuevos a los usados en épocas anteriores. No en vano, la digitalización de muchos de los procesos cotidianos desde finales del siglo XX y especialmente durante el siglo XXI demanda de los ciudadanos nuevas estrategias para gestionar su propio día a día. Y, desde luego, los procesos de aprendizaje no son una excepción a esto.

En este sentido, es frecuente partir de lugares comunes en esta reflexión, como las discusiones acerca de la naturaleza de los digital learners, en un intento de superar las antiguas dicotomías de nativos e inmigrantes digitales, por ejemplo. Desde luego, más allá de cuestionamientos casi de tipo antropológico sobre la esencia de las personas jóvenes en su relación con la tecnología, con la que conviven desde el nacimiento, surge la cuestión de cómo todo eso se capitaliza (o no) en términos de mejoras en el propio proceso de aprendizaje. Es también, por supuesto, un lugar común considerar que los nuevos estudiantes se enfrentan de una forma diferente a sus necesidades de aprendizaje. Y parecería lógico deducir de todo ello que, puesto que son nativos digitales, aprenden también digitalmente de modo eficiente. Con todo, acaso la intuición no sea tan certera y sea preciso conceptualizar de modo avanzado qué es esa competencia digital que los estudiantes universitarios precisan para maximizar sus oportunidades de aprendizaje no solo en la etapa universitaria, sino a lo largo de toda su vida. $\mathrm{Y}$, en consecuencia, es seguro que será productivo impulsar investigaciones que tomen este concepto como centro y que traten de operativizarlo para garantizar su adquisición.

Del mismo modo, los que asumimos el reto de la formación de los futuros maestros, cada vez más tomamos en consideración que los docentes del futuro (un futuro que es ya en parte presente) deben ser competentes también en el uso educativo de las tecnologías que tengan a su disposición. Y eso ya no es una derivada del concepto de competencia digital (del ciudadano, o del estudiante universitario a lo largo de su vida), sino un conjunto nuevo de destrezas y de conocimientos que debe garantizar su excelencia en el ejercicio profesional. También a estas reflexiones deben dedicarse los esfuerzos en el ámbito de la investigación en Tecnología Educativa, a fin de garantizar que los docentes pre-service (o in-service) devienen profesionales competentes de la educación.

El objetivo de la presente reflexión es, pues, presentar de forma sintética los avances de la investigación hasta el momento sobre estos dos conceptos, así como apuntar las líneas de investigación fututas que deberán seguirse para avanzar en este ámbito de conocimiento.

\section{MÉTODO}

En este contexto inicial que hemos expuesto de forma sucinta en el epígrafe introductorio precedente, adoptamos una metodología cualitativa orientada a la revisión y el análisis de la documentación relevante. Ese análisis documental parte de una selección de textos de la literatura del ámbito llevada a cabo por medio de búsquedas selectivas cruzadas de documentos a partir de las palabras clave relevantes en los repositorios más accesibles y sensibles a la temática (WoK, iCercador, Google Schoolar, ERIC, Scopus), lo cual nos ha permitido constituir una batería de textos con los que armar la presente reflexión. Este análisis documental ha seguido las fases descritas por Bisquerra (2004, p. 349) y Hernández, Fernández y Baptista (1991). 
Tras las lecturas iniciales, se procedió a organizar un mapa conceptual por medio del programa de análisis cualitativo de datos Atlas-Ti, a partir del cual se produjo la redacción de esta reflexión, que tiene por objetivos los siguientes:

- Aislar los conceptos de competencia digital y competencia digital docente y sus definiciones más relevantes para el contexto universitario español.

- Analizar el estado de la investigación educativa sobre estos dos tópicos, a fin de identificar las líneas futuras y las vías de oportunidad para los investigadores del ámbito.

\section{COMPETENCIA DIGITAL}

La reflexión sobre la competencia digital toma su origen en una nueva visión del aprendizaje en los estudios formales que parte de la necesidad de aislar aquel conjunto de destrezas y conocimientos que el propio individuo debe adquirir y consolidar como medio imprescindible para avanzar en sus estudios (en cualquier etapa formal, y a lo largo de la vida). Este conjunto, denominado competencias clave de forma genérica, se ha desarrollado en el ámbito universitario con especial profundidad, al menos en el discurso teórico, con el proceso de convergencia al Espacio Europeo de Educación Superior (EEES) que han venido experimentando en los últimos años las universidades españolas. Entre ellas, sin duda, la competencia digital ocupa una posición indiscutida, pues se considera que en la Sociedad de la Información es imprescindible contar con nuevas habilidades técnicas y cognitivas para hacer frente a los retos de conocimiento que se plantean y que, por ello, la competencia digital adquiere un rol protagonista en todos los aspectos del aprendizaje a lo largo de la vida (Selwyn, 2013). En ese sentido se han pronunciado también la mayoría de las instituciones de ámbito internacional. Y así, por ejemplo, la nómina de las competencias clave que propone la Comisión Europea (European Comission, 2007) también tiene en consideración la digital como una de las más relevantes; $y$, por su parte, el Consorcio para las $21^{\text {st }}$ Century Skills explicita que uno de los cuatro ámbitos competenciales importantes para enfrentarse a los retos de la SI es aquel que tiene que ver con las destrezas relativas a la información, a los medios y a la tecnología (P21, 2007).

En lo que no existe un consenso tan unánime es en la definición de la propia competencia digital (que, de hecho, se reconoce en diferentes denominaciones en el ámbito internacional, como este mismo, competencia digital, o digital competence, o digital literacy, o digital skills, o $21^{\text {st }}$ skills, entre otros, sin que lleguen a ser completamente sinónimos en todos los casos, ciertamente). En ese sentido, Gisbert y Esteve (2011) señalan la especial complejidad de poner orden en esta disparidad conceptual en relación directa con la complejidad del contexto digital al que debe servir, y señalan que se trata de un conjunto de herramientas, conocimientos y actitudes en los ámbitos tecnológico, comunicativo, mediático e informacional que configuran una alfabetización compleja y múltiple. En efecto, esta idea de complejidad y multiplicidad también se recoge en la propuesta de Ferrari (2013), que desarrolla el marco de referencia de la Comisión Europea, basado en una propuesta de 5 áreas (información, comunicación, creación de contenido, seguridad y resolución de problemas) que se concreta en 21 competencias necesarias para ser un usuario competente en entornos digitales. $\mathrm{Y}$, de modo paralelo, la UNESCO (2011), y en relación directa con su necesaria aplicación al ámbito educativo, propone su propio marco de referencia sobre la competencia digital en virtud del cual se establecen tres niveles de adquisición que evidencian el concepto complejo y estratificado subyacente: una primera fase de alfabetización digital, que permite a los estudiantes ser más eficientes en el manejo de la tecnología; una segunda fase de profundización en el conocimiento, que les permite profundizar mejor en el conocimiento de los diferentes ámbitos disciplinares; y una tercera fase de creación de conocimiento, en el que el manejo eficiente de la tecnología permite la creación de nuevo conocimiento a partir del anterior.

Ante esta disparidad de conceptualizaciones, de concreciones y de marcos de referencia, las universidades españolas han respondido de forma unánime en la consideración de que la competencia digital es una competencia nuclear indispensable, de primer orden, para el 
estudiante universitario; y de forma variada sobre los planteamientos prácticos de cómo trasladarla de modo efectivo a los currículos y a los planes de estudio. En este sentido, como decíamos, el EEES ha permitido poner sobre la mesa de la mayoría de las universidades la necesidad de abordarla explícita o implícitamente en todos los grados y posgrados universitarios. Y así, siguiendo a Larraz (2013), parece común considerar que el estudiante universitario deberá ser capaz de movilizar un conjunto de actitudes, destrezas y conocimientos digitales para resolver los problemas complejos que le plantea la SI. Algunas universidades, por un lado, han abordado específicamente la competencia digital diseñando materias que la atacan de forma directa; otras, por el otro, han planteado la necesidad de integrarla de forma transversal a lo largo de los estudios universitarios, a fin de garantizar que el alumnado la adquiere de forma efectiva al finalizar sus estudios de grado en un nivel suficiente. Sin embargo, esta consideración, que parece obvia, presenta más enjundia de la que parece, pues una parte importante de los esfuerzos de investigación se ha dedicado tanto a la conceptualización de ese suficiente, como al diseño de las estrategias de medición del nivel de la competencia digital.

En relación con lo primero, por ejemplo, en nuestro ámbito geográfico debemos partir indefectiblemente de la aproximación al concepto de competencia digital de Larraz (2013), quien no solo descompone operativamente el concepto en diferentes alfabetizaciones (multimedia, informacional, mediática y comunicativa), sino que presenta como resultado de su investigación una rúbrica completa, compleja y validada para evaluar la competencia digital. Lo primero en sí es especialmente relevante, por cuanto se trata de una propuesta de concepto aglutinadora, que integra las diferentes perspectivas hasta la fecha en una definición operativa, que puede ser aplicada directamente a ulteriores fases de la investigación al respecto; lo segundo, desde luego, es un paso más en la conceptualización, puesto que concreta más aún el concepto. Pero, además, supone una primera herramienta de evaluación de la competencia digital ya probada que cumple con todas las bondades de la rúbrica: determina los niveles de adquisición, dota de transparencia el proceso de evaluación, orienta el diseño de las situaciones de aprendizaje y las tutoriza. Ni que decir tiene que supone un gran avance en la investigación que deberá ser continuado con su generalización, con el análisis de las situaciones en que se aplique, etc. Son todo ello nuevas líneas de investigación que a buen seguro deberemos cubrir en el futuro para continuar completando esta parcela de conocimiento.

Otra de las ocupaciones de la investigación en nuestro ámbito geográfico en este sentido tiene que ver con el desarrollo de instrumentos de medición que automaticen la determinación del nivel de competencia del individuo, tanto a efectos de diagnóstico como de certificación. En el segundo ámbito, nos queda un largo camino por recorrer, pues se ha explorado poco cuáles pueden ser las estrategias más efectivas para certificar la competencia digital de los estudiantes universitarios (más allá de certificaciones elementales de determinados niveles de alfabetización tecnológica). En el primero, desde luego, hay algo más avanzado. En la Universitat Rovira i Virgili, se procedió hace unos años al diseño y a la validación de un instrumento de autopercepción de la competencia digital del alumnado universitario que ha gozado de abundante generalización hasta la fecha. Desde su presentación en 2011, INCOTIC (Gisbert, Espuny y González, 2011; González, Espuny y Gisbert, 2010) ha sido aplicado con éxito en muy diferentes contextos tanto en España como en Latinoamérica, siempre al servicio de procesos de diagnosis del nivel inicial de la competencia digital de los estudiantes universitarios al dar sus primeros pasos en la universidad; y se ha mostrado especialmente útil en la caracterización digital de las cohortes, lo cual es muy provechoso en el diseño específico de estrategias de aprendizaje. INCOTIC está actualmente en proceso de revisión, especialmente por lo que se refiere a la parte específica de autopercepción de las cuatro alfabetizaciones de la competencia digital ya señaladas (las secciones de uso y hábitos digitales permanecerán esencialmente iguales) y se espera que en breve se disponga ya de una versión validada que no solo permita su análisis de contextos concretos, sino la centralización de los resultados de aplicación para su estudio conjunto, lo cual puede ser muy interesante también a efectos de investigación.

De forma paralela, INCOTIC cuenta con una versión diseñada para los estudiantes de Secundaria Obligatoria, INCOTIC-ESO (Espuny, González y Gisbert, 2011). Este instrumento ha sido usado con profusión en el contexto español y se espera que se complemente con 
versiones que permitan hacer frente con garantías a la caracterización inicial de la competencia digital de los diferentes niveles educativos, aspecto capital en la Escuela 2.0.

\section{COMPETENCIA DIGITAL DOCENTE}

Como decíamos al inicio, otra de las cuestiones conectadas con la competencia digital de los estudiantes es la competencia digital de los profesores que deben acompañar a ese alumnado en su formación en la SI. Como señala Esteve (2015), no es suficiente con que los estudiantes de Educación adquieran su propia competencia digital, pues ello no garantiza que desarrollen las destrezas necesarias para diseñar estrategias de aprendizaje y acompañar al alumnado en su propio proceso de adquisición competencial. En este sentido, tiene mucho que ver el cambio de paradigma operado en las últimas décadas en torno al rol del docente, que ya no se limita a ser un mero transmisor de la información sino que ha devenido un guía o mentor, bajo el paraguas de los diferentes modelos de corte socioconstructivista de generación del conocimiento.

En este sentido, el docente que debemos formar en las facultades de Educación (lo que comúnmente se denomina pre-service teacher o teacher candidate) deberá enfrentarse en su ejercicio profesional a multitud de tareas simples o complejas que tienen que ver con la planificación, la intervención, la comunicación o la propia actualización (Carrera y Coiduras, 2012); y en todos esos ámbitos deberá ser competente en el uso de la tecnología. No en vano, no podemos concebir un docente de nuestro siglo que no considere el uso de las Tecnologías de la Información y la Comunicación (TIC) o que, en su aplicación, no exhiba criterios de excelencia; como tampoco podemos concebir un docente incapaz de mantener sus conocimientos actualizados, no solo en relación con su ámbito disciplinar, sino con el uso de cualesquiera tecnologías el futuro nos depare. A todo ello nos referimos cuando hablamos de la competencia digital docente, sean cuales sean las etiquetas que usemos para ello.

Abundando en estas ideas, por ejemplo, Krumsvik (2008) asevera que un uso básico de las TIC por parte de los docentes no es garantía de un ejercicio profesional competente, lo cual nos pone en la senda de considerar que el discurso sobre la tecnología educativa no puede deslindarse de otros aspectos capitales, como el criterio pedagógico o el contexto educativo de aplicación. Y en ese sentido, se aboga por dar un paso más en la formación de los docentes, que demasiado a menudo han visto hasta la fecha como la formación que recibían sobre TIC era genérica, técnica u orientada en exceso a la alfabetización más elemental, desligada del ámbito disciplinar y de la reflexión pedagógica o didáctica.

Si partimos de que, en la acción, la tecnología se debe usar en situaciones complejas, es natural pensar que un conjunto de destrezas tecnológicas no puede ser suficiente para el docente, sino que debemos abogar más por entender que la competencia digital docente viene conformada por las habilidades, las actitudes y los conocimientos requeridos por los educadores para apoyar el aprendizaje del alumno en el actual mundo digital (Hall, Atkins, y Fraser, 2014).

Otra cuestión, aún más compleja, tiene que ver con cómo operativizar estas conceptualizaciones de la competencia digital docente en propuestas prácticas que aplicar a la formación de maestros (o a la actualización de los maestros en ejercicio, como parte de su formación conjunta). En este sentido, uno de los modelos de referencia, sin duda, es el modelo TPACK (Koehler y Mishra, 2008), que considera que solo serán competentes los docentes que puedan activar de modo efectivo un conocimiento de triple naturaleza: disciplinar, pedagógico y tecnológico. No es solo imprescindible ser un experto en la materia o ámbito de conocimiento en el que se quiere formar a los alumnos (conocimiento del contenido), sino que se debe ser competente en la planificación de las estrategias didácticas más eficaces para cada tipo de competencia, conocimiento o destreza (conocimiento didáctico o pedagógico); y se deben dominar los recursos tecnológicos que potencian dichos aprendizajes (conocimiento tecnológico). Solo esa triple interacción nos lleva al ejercicio competente de la docencia; y, por tanto, será bajo ese prisma bajo el que deberemos formar a nuestros futuros maestros. Con idéntica intención, aunque con diferente enfoque, Krumsvik (2009) propone un modelo en capas sucesivas: en primer lugar, las habilidades básicas con TIC; en segundo lugar, la 
competencia didáctica con TIC; en tercer lugar, las estrategias de aprendizaje; y en cuarto lugar, cuando la integración de las capas anteriores es correcta, podremos hablar de competencia digital docente.

Paralelamente a esta reflexión teórica, y como consecuencia parcial de las necesidades inmediatas de hacer frente a la formación de futuros maestros, son diferentes los estándares o modelos de referencia que han definido diferentes instituciones y que debemos tomar en consideración. En primer lugar, la International Society for Technology in Education (ISTE, 2009) ha venido desarrollando los National Educational Technology Standards for Teachers (NETS-T), que especifican cómo deben ser las condiciones para una adecuada integración de la tecnología en la educación, así como los estándares que deben orientar el desarrollo profesional de los docentes, por medio de la rúbrica de evaluación que representan dichos estándares. Por otro lado, como decíamos anteriormente, la UNESCO (2008) ofrece su propio modelo de competencia digital con implicaciones en la competencia digital docente: además de los tres niveles o factores de dominio digital (alfabetización tecnológica, profundización en los conocimientos y creación de conocimiento) considera la existencia de 18 estándares o módulos que deben garantizar un desempeño docente competente en el mundo digital.

En contexto específicamente británico, el proyecto DigiLit Leicester trata de brindar el apoyo educativo en el despliegue de las TIC especialmente en el nivel de secundaria obligatoria (Fraser, Atkins, y Hall, 2013), y considera tres grandes etapas, al final de las cuales podemos hablar de docentes digitalmente competentes: la investigación y la definición en torno a la alfabetización digital, la identificación de los niveles de competencia según la percepción de los propios docentes y, por último, el apoyo en el propio desarrollo profesional como docente. Por último, y ya en contexto chileno, el proyecto Enlaces (ENLACES, 2011) propone cinco dimensiones que corresponden a funciones esenciales que desempeña el docente en cuanto a la integración de las TIC: la pedagógica, la técnica (o tecnológica), la de gestión, la social (ética y legal) y, por último, el desarrollo profesional.

En el contexto español, y en directa relación con la trasferencia de competencias en Educación a las Comunidades Autónomas, son estas las que han ido asumiendo el reto de concretar la necesidad de capacitación del docente tanto por lo que respecta a su formación universitaria, como en los planes de estratégicos de formación del profesorado en activo. Así, por ejemplo, en Cataluña el Departament d'Ensenyament de la Generalitat de Catalunya ha ido proponiendo bajo diferentes aproximaciones una rúbrica modelo que permita orientar el proceso de evaluación de la competencia digital docente de los profesores pre-service e inservice. Y, en el proceso de definición final ha constituido diferentes grupos de trabajo a los que ha invitado a representantes de las Facultades de Educación que le permitan desarrollar de forma conjunta la estrategia de renovación de los planes de formación de maestros y profesores, en formación y en activo. En líneas generales, el resto de las Comunidades Autónomas está siguiendo procesos parecidos, que varían en el detalle de su operativa, pero que persiguen un mismo objetivo.

También en nuestro contexto, el catalán, se han sucedido los impulsos institucionales de las agencias gubernamentales para el impulso de la investigación y el desarrollo en este ámbito. Por ejemplo, el proyecto de Mejora de la Formación Inicial de maestros COMDID busca precisamente el diseño y la validación de una estrategia formativa para el desarrollo de la competencia digital docente del profesorado en formación.

La investigación en este ámbito, desde luego, está aún en fase embrionaria por lo que respecta a su conjunto; y, por tanto, es mucho lo que queda por recorrer, y mucho lo que se recorre magnis itineribus por las demandas de la propia realidad educativa. Sin embargo, la investigación aplicada sobre este tópico no solo está explorando la conceptualización y el desarrollo de las correspondientes rúbricas de evaluación. Así, por ejemplo, las investigaciones de Esteve (2015) se han dedicado a explorar con provecho las posibilidades de los entornos de simulación 3D para la evaluación de esta competencia en estudiantes universitarios de Educación, con el fin no solo de determinar su eficacia, sino también de ver vías de oportunidad en diferentes aspectos. Y, de forma más amplia, y como consecuencia parcial de estos avances, el proyecto Simul@b (http://late-dpedago.urv.cat/precercasimulab/), financiado por el Plan Estatal de I+D+i del Ministerio de Economía y Competitividad español, viene 
desarrollando un laboratorio 3D destinado precisamente a la adquisición de esta competencia por parte de maestros pre-service, con todo lo que ello conlleva: depuración y priorización de indicadores de la rúbrica, el diseño de actividades de adquisición y consolidación de la competencia y su posterior evaluación. Aunque radicado en el contexto de las universidades catalanas, en el ánimo del proyecto está que pueda generalizarse a diferentes realidades universitarias con leves adaptaciones y que del proceso de I+D se obtengan principios de diseño altamente útiles en el desarrollo de otras estrategias similares o en la investigación acerca de los posibles instrumentos o estrategias que puedan usarse en la acreditación de la competencia digital docente.

\section{NUEVAS LÍNEAS DE INVESTIGACIÓN Y CONCLUSIONES}

En los dos capítulos anteriores hemos tratado de ofrecer una panorámica sintética de la investigación aplicada en el ámbito de la Tecnología Educativa en relación con los conceptos de competencia digital y competencia digital docente, que son los que más cercanos tenemos a nuestro perfil como investigadores. Sin duda, es mucho lo que se ha avanzado en los últimos años a este respecto. $Y$ eso es especialmente relevante, si tenemos en cuenta que la investigación ha debido avanzar en ocasiones por delante de la conceptualización, para dar respuesta a las diferentes necesidades que iba planteando la realidad educativa. Sin embargo, es mucho lo que queda por avanzar en relación con ambos conceptos, y es a ello a lo que nos dedicaremos a continuación, a fin de estimular la adhesión de cualquier investigador a este apasionante ámbito de conocimiento.

Por lo que respecta a la competencia digital, partimos de una situación favorable, como decíamos, en la que contamos con una rúbrica aplicable e instrumentos de diagnóstico vigentes o en proceso de revisión cercana. Con todo, es preciso avanzar en dos grandes ámbitos de manera prioritaria. El primero tiene que ver con el diseño de estrategias de aprendizaje estandarizadas que garanticen la adquisición de la competencia digital del alumnado universitario. Es indudable que las universidades han ido dando respuesta efectiva a esta necesidad desde la implantación de los grados EEES; sin embargo, es importante avanzar en la estandarización de esas respuestas y en la investigación acerca de su eficacia, a fin de diseñar estrategias conjuntas productivas mejores, que contribuyan a un mejor proceso de adquisición competencial. Asimismo, es preciso agilizar y operativizar el proceso de evaluación final de la competencia digital en los diferentes niveles educativos. En la medida en que las diversas instituciones estamos obligadas a acreditar niveles de adquisición de nuestro alumnado, deberíamos contar con instrumentos que ayuden a estandarizar este proceso y, en la medida de lo posible, a hacerlo más automático y más transparente. Los instrumentos de diagnóstico disponibles hasta el momento son una orientación, y pueden ser tomados como referencia; pero deben ser ampliados convenientemente para que sirvan para los procesos de certificación y acreditación oficiales.

En cuanto a la competencia digital docente, como también hemos visto, es bastante el camino avanzado, tanto en la conceptualización como en el diseño de estrategias de aprendizaje ligadas a esta competencia; y son prometedores los avances que las administraciones y las universidades y sus grupos de investigación están realizando al respecto. Con todo, también es posible identificar interesantes e importantes líneas de investigación que deberán desarrollarse en el futuro inmediato y que tienen que ver, en efecto, con las dos anteriores que hemos referido para la competencia digital del estudiante universitario. Por un lado, y en relación con la necesidad de las autoridades educativas de permitir al profesorado la acreditación de la competencia digital docente (como requisito para el ejercicio profesional o como parte de la carrera profesional), está el desarrollo de instrumentos que permitan acreditar dicha competencia y que, en la medida de lo posible, contribuyan a un proceso de certificación transparente, global y eficaz desde el punto del uso de recursos materiales, temporales y humanos. En ese sentido, los avances del proyecto Simul@b guiarán mucho la investigación en este sentido, al ofrecernos evidencias de las potencialidades de los entornos de simulación 3D (la tecnología avanzada) en el momento de la evaluación.

Por otro lado, también es imperativo definir la estrategia de aprendizaje que garantizará que los futuros maestros y los docentes en activo adquieren esta competencia. Esta vía de 
investigación, ligada a los presupuestos de la investigación para el diseño, debe ofrecer modelos formativos eficaces y generalizables, que tengan el respaldo de las rúbricas y los estándares de referencia y que garanticen unos mínimos máximos para cada uno de los colectivos.

Como se ve, la investigación aplicada en el ámbito de la Tecnología Educativa no va solo ligada al análisis exploratorio o comprobatorio de las potencialidades educativas de determinados recursos. En nuestro caso, la aproximación es más pedagógica y mucho más transversal, y tiene en cuenta cuáles son las competencias que necesitan los individuos, en su rol de estudiantes o de docentes, para usar de manera eficaz cualesquiera tecnologías a las que tengan acceso. Son investigaciones de largo alcance y, por supuesto, de mucho impacto en nuestros contextos educativos. $Y$ en ellas, aunque ya es considerable lo que se que avanzado, aún queda mucho por recorrer si queremos maximizar la incidencia en el proceso de enseñanza-aprendizaje, tanto por parte de quienes diseñan las estrategias docentes como de quienes las protagonizan.

\section{RECONOCIMIENTOS}

La presente investigación se ha llevado a cabo dentro del proyecto de investigación Simul@b. Laboratorio de simulaciones 3D para el desarrollo de la competencia digital docente (referencia EDU2013-42223-P), financiado por el Ministerio de Economía y Competitividad del Gobierno de España, en su Plan Estatal de I+D+i, convocatoria de 2013.

\section{REFERENCIAS BIBLIOGRÁFICAS}

Bisquerra, R. (2004). Metodología de la Investigación Educativa (2. a edició.). Madrid: La Muralla.

Carrera, F. X., y Coiduras, J. L. (2012). Identificación de la competencia digital del profesor universitario: Un estudio exploratorio en el ámbito de las ciencias sociales. Revista de Docencia Universitaria (REDU), 10(2), 273-298.

ENLACES. (2011). Competencias y estándares TIC para la profesión docente. Santiago de Chile.

Espuny, C., González, J., y Gisbert, M. (2011). INCOTIC-ESO. ¿Cómo radiografiar la competencia digital del alumnado en el programa eduCAT1x1? In G. de C. y Educación y U. A. de Barcelona (Eds.), Estrategias de alfabetización mediática: Reflexiones sobre comunicación y educación (pp. 1-7). Barcelona: UAB.

Esteve, F. (2015). La competencia digital docente. Análisis de la autopercepción y evaluación del desempeño de los estudiantes universitarios de Educación por medio de un entorno 3D. Universitat Rovira i Virgili.

European Comission, E. (2007). Key competences for lifeling learning. Brussels.

Ferrari, A. (2013). DIGCOMP. A framework for Developing and Understanding Digitas/ Competence in Europe. Sevilla.

Fraser, J., Atkins, L., y Hall, R. (2013). DigiLit leicester. Supporting teachers, promoting digital literacy, transforming learning. Leicester: Leicester City Council.

Gisbert, M., Espuny, C., y González, J. (2011). INCOTIC. Una herramienta para la @utoevaluación diagnóstica de la competencia digital de la universidad. Profesorado. Revista de Currículum Y Formación Del Profesorado, 15(1), 75-90.

Gisbert, M., y Esteve, F. M. (2011). Digital learners: la competencia digital de los estudiantes universitarios. La Cuestión Universitaria, 7, 48-59. 
González, J., Espuny, C., y Gisbert, M. (2010). La evaluación cero de la competencia nuclear digital en los nuevos grados del EEES. @tic. Revista D'innovació Educativa, 4, 13-20.

Hall, R., Atkins, L., y Fraser, J. (2014). Defining a Self-Evaluation Digital Literacy for Secondary Educators. the DigiLit Leicester Project. Research in Learning Technology, 22, 21440. doi:http://dx.doi.org/10.3402/rlt.v22.21440

Hernández, R., Fernández, C.,y Baptista, P. (1991). Metodología de la Investigación. Ciudad de México: Mc Graw-Hill.

ISTE. (2009). National educational technology standards for teachers. Washington, DC.

Koehler, M. J., y Mishra, P. (2008). Introducing technological pedagogical content knowledge. In A. E. R. Association (Ed.), Annual meeting of the American Educational Research Association. Annual meeting of the american educational research association.

Krumsvik, R. J. (2008). Situated learning and teachers' digital competence. Education and Information Technologies, 13(13), 279-290. doi:doi:10.1007/s10639-008-9069-5

Krumsvik, R. J. (2009). Situated learning in the network society and the digitised school. European Journal of Teacher Education, 32(2), 167-185.

Larraz, V. (2013). La competència digital a la Universitat. Universitat d'Andorra.

P21. (2007). Framework fo 21st century learning. Tucson (Arizona).

Selwyn, N. (2013). Education in a Digital World: Global Perspectives on Technology and Education. London: Routledge.

UNESCO. (2008). Estándares de competencia TIC para docentes. París.

UNESCO. (2011). UNESCO ICT Competency Framework for Teachers. Paris.

\section{INFORMACIÓN SOBRE LOS AUTORES}

\section{Mercé Gisbert Cervera}

Universitat Rovira i Virgili

Profesora Titular del área de Didáctica y Organización Escolar del departamento de Pedagogía de la Universidad Rovira i Virgili de Tarragona. Ha desempeñado diversos cargos de gestión en la URV: Vice-Decana de la Facultad de Ciencias de la Educación y Psicología (1994- 1997), directora del Instituto de Ciencias de la Educación (1998-2001), directora del Servicio de Recursos Educativos (de 2001 a 2002) y Vicerrectora de Política Docente y Convergencia del EEES (2002-2010). Coordina el Doctorado Interuniversitario a distancia en Tecnología Educativa (UdL, UM, UIB, URV). Es Investigadora principal de ARGET: Applied Research Group in Education and Technology (ref. 2009SGR596), sus líneas de investigación se enmarcan en las TIC aplicadas a la educación. Ha coordinado diversos proyectos de investigación relacionados con éste ámbito tales como GET y Pupitre (MCYT), DUCANET (MECD), SIMUL@ (Plan Nacional de I+D+I 2008-2011), y SIMUL@B (Plan Nacional de I+D+i 2014-2017) que tienen centrados en el estudio de las competencias transversales y la competencia digital docente así como la participación en el proyecto Igualdader, proyecto de la Iniciativa Comunitaria EQUAL del Fondo Social Europeo. Ha sido miembro del 2011-2015 del Consejo Escolar de Catalunya. Actualmente es miembro del Consejo Nacional de la Cultura y las Artes de Catalunya.

Web: http://late-dpedago.urv.cat/mgisbert 


\section{Juan González Martínez \\ Universitat Rovira i Virgili}

Profesor del Departament de Pedagogia de la Universitat Rovira. Doctor en Filología Hispánica (UNED) y en Tecnología Educativa (URV). Coordinador del Máster en Tecnología Educativa: eLearning y Gestión del Conocimiento de la URV. Miembro del grupo de investigación consolidado ARGET (Applied Research Group in Education and Technology), con identificador 2014SGR1399. Investigador del proyecto Simul@b. Laboratorio de simulaciones 3d para el desarrollo de la competencia digital docente, financiado por el Ministerio de Economía y Competitividad (ref. EDU2013-42223-P). Su principal línea de investigación tiene que ver con la competencia digital (concepto e instrumentos para su medición en los niveles educativos de Secundaria y Universidad) y la competencia digital docente.

ResearcherlD: http://www.researcherid.com/rid/E-9480-2015

ResearchGate: https://www.researchgate.net/profile/Juan_Gonzalez_Martinez

Web: http://late-dpedago.urv.cat/jgonzalez/

\section{Francesc Esteve Mon}

Universitat Rovira i Virgili

Es profesor e investigador postdoctoral en el Departamento de Pedagogía de la Universitat Rovira i Virgili (Tarragona). Imparte su docencia en los estudios de Educación Infantil y Primaria, Pedagogía y en el Máster Interuniversitario de Tecnología Educativa. Es doctor internacional en Tecnología Educativa, y miembro del grupo de investigación Applied Research Group in Education and Technology (ARGET). Centra su investigación en el análisis de la competencia digital, la formación inicial docente y en el estudio de la metodología de investigación basada en el diseño (design-based research). Visiting Scholar en la Universidad de Leiden (2014), ha trabajado como investigador en la Cátedra UNESCO de Gestión y Política Universitaria, en el Centro de Educación y Nuevas Tecnologías (CENT), y como evaluador en la Agencia Nacional de Evaluación de la Calidad y Acreditación (ANECA). Es autor de diferentes publicaciones relacionadas con el uso de las TIC en los procesos de enseñanzaaprendizaje y revisor de diferentes revistas internacionales.

Web: http://www.francescesteve.es

Twitter: https://twitter.com/francescesteve

\section{$(\mathrm{cc}) \mathrm{EYY}_{\mathrm{NC}}$}

Los textos publicados en esta revista están sujetos a una licencia de Reconocimiento 4.0 España de Creative Commons. Puede copiarlos, distribuirlos, comunicarlos públicamente y hacer obras derivadas siempre que reconozca los créditos de las obras (autoría, nombre de la revista, institución editora) de la manera especificada por los autores o por la revista. La licencia completa se puede consultar en:Licencia Creative Commons Atribución-NoComercial 4.0 Internacional. 